\title{
Particle transport in a moving corner
}

\author{
By F. E. LAINE-PEARSON ${ }^{1}$ AND P. E. HYDON \\ ${ }^{1}$ Department of Mathematics and Statistics, University of Surrey, Guildford GU2 7XH, UK \\ f.laine-pearson@surrey.ac.uk; p.hydon@surrey.ac.uk
}

(Received 8 June 2005 and in revised form 24 January 2006)

This paper describes particle transport in Stokes flow in a two-dimensional corner whose walls oscillate, which is a simple model for particle transport in the pulmonary alveoli. Formally speaking, the wall motion produces a perturbation to the wellknown Moffatt corner eddies. However, this 'perturbation' is dominant as the corner is approached. The motion of particles is regular near to the corner. Far from the corner, chaotic motion within the main part of the flow is restricted to very small regions. We deduce that there is competition between the far-field motion that generates eddies and the wall motion. The relative strengths of these two motions determines whether a given particle moves regularly or chaotically. Consequently, there is an intermediate region in which chaotic transport is maximized.

\section{Introduction}

This mathematical study is motivated by an ongoing investigation into particle motion in the lung, led by Tsuda, that combines physiological, mathematical and computational studies of chaos in alveoli. These are cavities in the lower airway walls in which recirculation can occur. Tsuda, Henry \& Butler (1995) examined the effects of cyclic expansion and contraction of alveolar walls on fluid flow in such a cavity by developing a numerical model. It was observed that low-Reynolds-number alveolar flow can be extremely complex; it was presumed that the alveolated duct structure and its time-dependent motion induced this complexity. In a related study, Haber et al. (2000) developed an analytical model of a cyclically expanding and contracting spherical alveolus and its vicinity. Their results supported the observation that there is a level of complexity of particle mixing in this region of the lungs. Moreover, the geometric features of structural alveolation and rhythmic expansion were given as the mechanism for chaotic mixing of particles. The numerical simulations of Henry, Butler \& Tsuda (2002) quantified the effects of cyclic expansion and contraction of an alveolated duct upon particle motion in the model alveoli. Lagrangian tracking of fluid particles indicated that the trajectories exhibit unpredictable stretched and folded patterns. These observations led Tsuda et al. (2002) to hypothesize that chaotic flow can occur in alveolated airways, and that this can result in flow-induced aerosol mixing and deposition deep in the lung. Tsuda's group tested this hypothesis by performing flow visualization experiments in excised animal lungs. They ventilated lungs with ultra-low-viscosity, polymerizable, Newtonian fluids of two colours. Each lung was first filled with white fluid, then ventilated with blue fluid for a number of cycles, ensuring that the Reynolds number remained low throughout the experiment. Then ventilation was halted and the fluids were polymerized to make casts that showed the final position of fluid particles. Recirculation had occurred in many alveoli, and there was substantial mixing of the fluids after just two cycles of inspiration and expiration. 
The current paper investigates the mechanism by which chaotic particle motion can occur in a two-dimensional cavity with recirculating fluid, when the cavity expands and contracts periodically. We restrict attention to Stokes flow, which is the flow regime that operates in the alveoli. Furthermore, it is assumed that particles move passively with the fluid. For boundary conditions, we chose a simple shape that admits recirculation, namely a corner region. This study aims to qualify the phenomena occurring when recirculating particles are affected by rhythmic wall motion and this will be achieved by drawing from the mathematical theory developed for Hamiltonian dynamical systems.

Corner flows - and their three-dimensional counterparts - have been considered in the literature by many authors, including Moffatt (1964), Liu \& Joseph (1978), Weidman \& Calmidi (1999), Shankar (2000), Chetan, Weidman \& Davis (2005). The seminal work on Stokes flow in a corner is Moffatt (1964), which demonstrated that a sequence of eddies can be generated by a flow past the corner, provided that the angle between the walls of the corner does not exceed $2 \phi_{\text {critical }} \approx 146.3^{\circ}$. As the corner is approached, successive eddies are of decreasing size and greatly decreasing intensity. Each eddy is at least 350 times weaker than its larger neighbour.

Corner eddies can be generated in a triangular container of viscous fluid when a cylinder is rotated anywhere inside it. Moffatt hypothesized that it should be straightforward to observe one eddy, but that to observe a sequence of them might present insurmountable experimental difficulties. Fifteen years later, Taneda (1979) realized two eddies in succession.

The purpose of the current paper is to investigate the qualitative nature of particle transport when an externally driven antisymmetric Stokes flow in a corner of angle less than $2 \phi_{\text {critical }}$ is perturbed by wall motion. The flow field is described in $\S 2$. In $\S 3$, the equations of motion for a passive particle are written as a Hamiltonian system. This formulation plays an important part in revealing the behaviour of particle trajectories, and gives some insight into the requirements for the breakup of recirculating particle paths, as discussed in $\S 4$.

\section{The flow field}

\subsection{Steady flow in the unperturbed corner}

Throughout the paper, we use carets to denote dimensional variables; these are removed when variables are non-dimensionalized.

Moffatt (1964) considered two-dimensional Stokes flow in a rigid corner, which is induced by an arbitrary flow past the corner at a large distance. In plane polar coordinates $(\hat{r}, \theta)$, the Stokes equation for the streamfunction $\hat{\psi}(\hat{r}, \theta)$, namely $\nabla^{4} \hat{\psi}=0$, admits separated solutions of the form $\hat{\psi}=\operatorname{Re}\left\{\hat{r}^{\lambda_{0}} f\left(\theta ; \lambda_{0}\right)\right\}$. If the walls are at $\theta= \pm \phi_{0}$ then the boundary conditions are

$$
\hat{\psi}_{, \hat{r}}=\hat{\psi}_{,}=0 \quad \text { at } \quad \theta= \pm \phi_{0}
$$

the comma denotes partial differentiation. For antisymmetric flow with $\lambda_{0} \notin\{0,1,2\}$, which occurs in corner eddies,

$$
f\left(\theta ; \lambda_{0}\right)=K_{0}\left[\cos \left(\left(\lambda_{0}-2\right) \phi_{0}\right) \cos \left(\lambda_{0} \theta\right)-\cos \left(\lambda_{0} \phi_{0}\right) \cos \left(\left(\lambda_{0}-2\right) \theta\right)\right],
$$

where $K_{0}$ is a complex constant that is determined by conditions far from the corner. The eigenvalues $\lambda_{0}$ are related to the angle $\phi_{0}$ by

$$
\left(\lambda_{0}-2\right) \tan \left(\left(\lambda_{0}-2\right) \phi_{0}\right)=\lambda_{0} \tan \left(\lambda_{0} \phi_{0}\right),
$$


which is trigonometrically equivalent to

$$
\sin \left(2 \phi_{0} \mu_{0}\right)=-\mu_{0} \sin \left(2 \phi_{0}\right), \quad \text { where } \quad \mu_{0}=\lambda_{0}-1 .
$$

Moffatt showed that every eigenvalue is complex if $\phi_{0}<\phi_{\text {critical }}$, and thus the flow is a sequence of eddies. Following Moffatt, we consider only the flow corresponding to the dominant eigenvalues $\lambda_{0}$ and $\overline{\lambda_{0}}$ (in other words, the pair of eigenvalues with smallest positive real part). Here

$$
\lambda_{0}=\left(1+\frac{\xi}{2 \phi_{0}}\right)+\mathrm{i}\left(\frac{\eta}{2 \phi_{0}}\right),
$$

where $\xi \approx 4$ and $\eta$ is $O(1)$. Note that as $\phi_{0}$ tends to zero, $\lambda_{0 R} \equiv \operatorname{Re}\left\{\lambda_{0}\right\}$ grows rapidly. The range of values of $\hat{r}$ for which the Stokes approximation is valid is given by the condition

$$
R e=\frac{\mathscr{D} \hat{r}^{\lambda_{0 R}}}{v} \ll 1,
$$

where $R e$ is the Reynolds number based on distance from the corner, $v$ is the kinematic viscosity of the fluid, and $\mathscr{D}$ is a dimensional constant such that $f\left(\theta ; \lambda_{0}\right) / \mathscr{D}$ is of order unity. As $\lambda_{0 R}$ is positive, inertial forces are negligible for sufficiently small $\hat{r}$. From here on, such a flow is referred to as 'Moffatt flow'. Figure 1 illustrates typical particle paths in Moffatt flow. The initial conditions are spaced out along the line $y=0$. The particles on the right-hand eddy move clockwise, while the particles on the left-hand eddy move anticlockwise. Consequently, a separatrix lies between these two eddies; it is a curved line that is attached to each wall. Moffatt flow has an infinite sequence of eddies in the corner, which have been generated by the far-field flow. Each eddy lies in its own cell, whose boundaries are the walls of the corner and a separatrix on either side. The pair of eddies of figure 1 has been chosen specifically so that the two distinct types of behaviour that appear when the walls are moved can be clearly observed (see later figures).

\subsection{Quasi-steady flow}

Now consider what happens when the steady flow is perturbed by a periodic wall motion that keeps the maximum angle of the corner less than $2 \phi_{\text {critical }}$. Let $\hat{\boldsymbol{u}}=\hat{u}_{r} \boldsymbol{e}_{r}+$ $\hat{u}_{\theta} \boldsymbol{e}_{\theta}$ be the velocity field with respect to the steady frame coordinates $(\hat{r}, \theta)$. The incompressibility condition in cylindrical polar coordinates is

$$
\frac{1}{\hat{r}}\left(\hat{r} \hat{u}_{r}\right)_{\hat{r}}+\frac{1}{\hat{r}}\left(\hat{u}_{\theta}\right)_{, \theta}=0 .
$$

As the corner region is simply connected, there exists a streamfunction $\hat{\psi}(\hat{r}, \theta, \hat{t})$ such that $\hat{u}_{r}=\hat{\psi},{ }_{\theta} / \hat{r}$ and $\hat{u}_{\theta}=-\hat{\psi}, \hat{r}$. Therefore particle motion is described by the equations

$$
\frac{\mathrm{d} \hat{r}}{\mathrm{~d} \hat{t}}=\frac{1}{\hat{r}} \hat{\psi},{ }_{\theta}, \quad \hat{r} \frac{\mathrm{d} \theta}{\mathrm{d} \hat{t}}=-\hat{\psi}, \hat{r} .
$$

The walls are at $\theta= \pm \phi$, where

$$
\phi=\phi_{0}[1+\epsilon \sin (\omega \hat{t})]
$$

here $\omega$ is the frequency of oscillation and $0 \leqslant \epsilon<1$. Therefore the boundary conditions are

$$
\hat{\psi}_{\theta}=0, \quad \hat{\psi}, \hat{r}=\mp \epsilon \phi_{0} \omega \hat{r} \cos (\omega \hat{t}) \quad \text { on } \quad \theta= \pm \phi .
$$



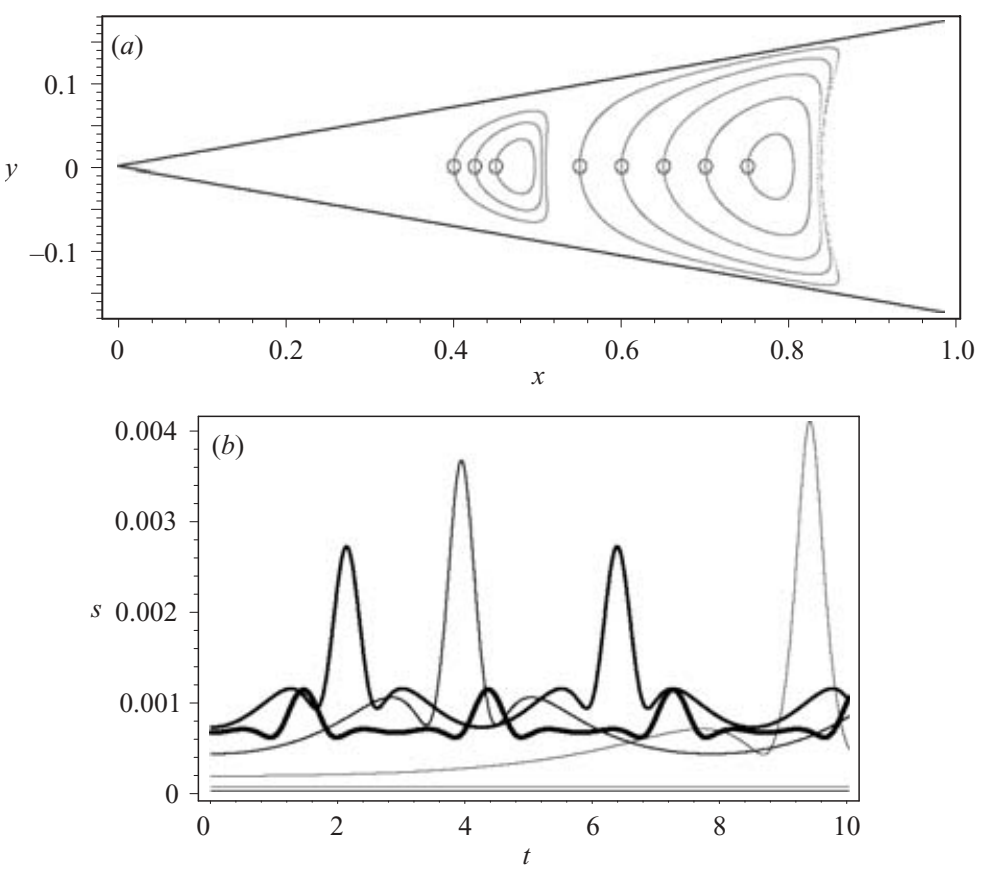

Figure 1. (a) Moffatt flow when $2 \phi_{0}=20^{\circ}$ and $K_{0}=1 / 2 \pi$. Eight initial conditions along $y=0$ (each marked by a circle) have been evolved; each trajectory is composed of 2000 points. Two consecutive eddies are shown. As the flow is steady, particle paths coincide with streamlines (see Acheson 1990). (b) The speed $(s)$ of the eight particles of Moffatt flow when $2 \phi_{0}=20^{\circ}$ and $K_{0}=1 / 2 \pi$ are tracked for $t=0 \ldots 10$, where $t$ relates to the 'non-dimensionalized time' of the quasi-steady flow. The highest five curves represent particles belonging to the right-hand eddy, while the lower line represents three particles in the left-hand eddy (the three curves here coincide at this scale). Scanning from left to right of $(a)$, and reading from bottom to top of (b), the periods of the particles are approximately 1717, 1038, 731, 68.1, 18.7, 7.8, 4.2, 2.3 time units, respectively.

To non-dimensionalize the problem, let

$$
t=\frac{\omega \hat{t}}{2 \pi}, \quad r=\frac{\hat{r}}{a}, \quad \psi=\frac{\hat{\psi}}{a^{2} \omega},
$$

where $a$ is a convenient length scale. We discuss our choice of $a$ at the end of this section. Define $\alpha^{2}=a^{2} \omega / \nu$ to be the Womersley number. Provided that $\alpha^{2} / 2 \pi \ll 1$, unsteady inertia can be neglected and the flow is quasi-steady. Thus the combined flow is simply a superposition of Moffatt flow and the flow generated through wall motion alone (which we shall call the squeeze flow for brevity). Hence $\psi=\psi_{M}+\psi_{W}$, where $\psi_{M}$ is the non-dimensional Moffatt streamfunction for a corner whose walls are at $\theta= \pm \phi$, and where $\psi_{W}$ is the non-dimensional streamfunction associated with the flow driven by the wall motion. Specifically,

$$
\psi_{M}(r, \theta, t)=K \operatorname{Re}\left\{r^{\lambda}[\cos ((\lambda-2) \phi) \cos (\lambda \theta)-\cos (\lambda \phi) \cos ((\lambda-2) \theta)]\right\},
$$

where $\lambda$, which is time dependent, and $\phi$ satisfy

$$
(\lambda-2) \tan ((\lambda-2) \phi)=\lambda \tan (\lambda \phi) \text {. }
$$

As for the steady flow, only the dominant eigenvalues are considered. The amplitude $K$ depends on the far-field flow and is assumed to be a real constant for this investigation. 
The solution (2.2) satisfies $\psi_{M, \theta}=\psi_{M, r}=0$ on the walls. Therefore the non-zero boundary condition produced by the wall motion generates the squeeze flow

$$
\psi_{W}(r, \theta, t)=-\epsilon \phi_{0}\left(\frac{r^{2}}{2}\right) \cos (2 \pi t)\left[\frac{\sin (2 \theta)-2 \theta \cos (2 \phi)}{\sin (2 \phi)-2 \phi \cos (2 \phi)}\right],
$$

which is described in Moffatt (1964). According to Moffatt \& Duffy (1980), this describes the Stokes flow produced by wall motion when $2 \phi<\tan (2 \theta)$ and $r$ is not too large. Although the squeeze flow is unsteady, the acceleration terms in the NavierStokes equations are negligible in the region near the corner for which

$$
\left|\frac{\mathrm{d} \phi}{\mathrm{d} \hat{t}}\right| \frac{\hat{r}^{2}}{v} \ll 1, \quad \text { that is, } \quad \epsilon \phi_{0} \alpha^{2} r^{2} \ll 1 .
$$

We restrict attention to this region from here on, so that the combined flow is quasisteady. Convective inertia is negligible provided that $R e$ is sufficiently small.

The squeeze flow is integrable, which can be observed by numerically tracking particles during a half-cycle and noting that they faithfully retrace their paths during the next half-cycle. Moreover, particle motion in the squeeze flow satisfies

$$
\frac{1}{2 \pi} \frac{\mathrm{d} r}{\mathrm{~d} t}=\frac{1}{r} \frac{\partial \psi_{W}}{\partial \theta} \quad \text { and } \quad \frac{1}{2 \pi} \frac{\mathrm{d} \theta}{\mathrm{d} t}=-\frac{1}{r} \frac{\partial \psi_{W}}{\partial r} .
$$

Therefore

$$
\frac{\mathrm{d} r}{\mathrm{~d} \theta}=-\frac{r}{2}\left[\frac{2 \cos (2 \theta)-2 \cos (2 \phi)}{\sin (2 \theta)-2 \theta \cos (2 \phi)}\right]
$$

and

$$
\frac{\mathrm{d} \theta}{\mathrm{d} t}=2 \pi \epsilon \phi_{0} \cos (2 \pi t)\left[\frac{\sin (2 \theta)-2 \theta \cos (2 \phi)}{\sin (2 \phi)-2 \phi \cos (2 \phi)}\right] .
$$

As $\phi$ is a function of $t$ it follows that $\phi$ can be written as a function of $\theta$ by solving the first-order differential equation (2.6). Substituting this into equation (2.5) gives a first-order differential equation, which can be integrated (in principle) to obtain a first integral. The flow $\psi_{W}$ is integrable essentially because of the reversibility of steady Stokes flow. The adapted Moffatt flow (Moffatt flow for $\epsilon \neq 0$ ) is not integrable; moreover this flow cannot occur independently as it does not satisfy the moving boundary conditions.

Formally, the squeeze flow and the modification to the Moffatt flow caused by the wall motion combine to produce an $O(\epsilon)$ perturbation to the steady Moffatt flow. However, the steady Moffatt flow diminishes far more rapidly than the squeeze flow as the corner is approached. To a first approximation, for $2 \phi_{0}$ the decay is $O\left(r^{1+\xi / 2 \phi_{0}}\right)$ where $\xi \approx 4$. (For example, for $2 \phi_{0}=20^{\circ}$ the decay is $O\left(r^{13}\right)$, and for $2 \phi_{0}=60^{\circ}$ the decay is $O\left(r^{5}\right)$.) By contrast, the squeeze flow is $O\left(r^{2}\right)$. Consequently, for $r<1$, the 'perturbation' to the steady Moffatt flow has an increasingly great effect as $r \rightarrow 0$. For instance, in the right-hand eddy of figure 2, the perturbation is small in the central region, and has an increasing effect in the outer region where $r$ is smaller for much of the particle's motion.

There is no intrinsic length scale for the steady Moffatt flow. However, when the wall motion is added, the two motions are of comparable size only in a limited range of values of $\hat{r}$. Therefore the appropriate length scale $a$ is the order of magnitude of these values. In dimensionless terms this ensures that the two motions are comparable for $r=O(1)$. 


\section{Particle motion in the perturbed flow}

For any two-dimensional flow with a streamfunction $\psi(r, \theta, t)$ that is nondimensionalized as above, particle trajectories are obtained from the Lagrangian equations of motion

$$
\frac{1}{2 \pi} \frac{\mathrm{d} r}{\mathrm{~d} t}=\frac{1}{r} \frac{\partial \psi}{\partial \theta}, \quad \frac{1}{2 \pi} \frac{\mathrm{d} \theta}{\mathrm{d} t}=-\frac{1}{r} \frac{\partial \psi}{\partial r} .
$$

These equations can be written as a Hamiltonian system for $R=r^{2}$ and $\theta$, as follows:

$$
\frac{\mathrm{d} R}{\mathrm{~d} t}=-\frac{\partial H}{\partial \theta}, \quad \frac{\mathrm{d} \theta}{\mathrm{d} t}=\frac{\partial H}{\partial R},
$$

where the Hamiltonian $H$ is

$$
H(R, \theta, t)=-4 \pi \psi\left(R^{1 / 2}, \theta, t\right) .
$$

In particular, in the absence of wall motion, the Hamiltonian is

$$
H_{0}(R, \theta)=-4 \pi \lim _{\epsilon \rightarrow 0} \psi_{M}\left(R^{1 / 2}, \theta, t\right) \text {. }
$$

The system

$$
\frac{\mathrm{d} R}{\mathrm{~d} t}=-\frac{\partial H_{0}}{\partial \theta}, \quad \frac{\mathrm{d} \theta}{\mathrm{d} t}=\frac{\partial H_{0}}{\partial R},
$$

is integrable; $H_{0}$ is conserved during a particle's motion. Consequently there exist action-angle coordinates in terms of which the flow on each streamline is a steady rotation.

For the moving corner, the Hamiltonian is $H=H_{0}+H_{1}$, where

$$
H_{1}(R, \theta, t)=-4 \pi \psi_{M}\left(R^{1 / 2}, \theta, t\right)-4 \pi \psi_{W}\left(R^{1 / 2}, \theta, t\right)-H_{0}(R, \theta) .
$$

Note that $H_{1}$ vanishes as $\epsilon \rightarrow 0$. Formally, $H_{1}$ is of order $\epsilon$ provided that

$$
\lambda=\lambda_{0}+O(\epsilon) \text {. }
$$

The Hamiltonian $H$ has been constructed in this way so that ideas from KAM (Kolmogorov-Arnol'd-Moser) theory can be consulted. KAM theory is a methodology of how to approach certain problems in perturbation theory that are linked with small devisors. Although there are many variants of the KAM theorem, it is the classical KAM theorem, which is concerned with the stability of motions in Hamiltonian systems constructed from slightly perturbed integrable Hamiltonian systems, that will be referred to as the 'KAM theorem' here. For $H, H_{0}$ is the integrable part, which is essentially $\psi_{M}$ evaluated at $\epsilon=0$, while $H_{1}$ is some non-integrable perturbation, which accounts for the additional terms generated by the perturbation parameter $\epsilon$ through $\psi_{W}$ and the compensation of $\psi_{M}$ to $\epsilon \neq 0$. An assumption of the theory is that $\epsilon \ll 1$.

The KAM theorem implies that, if $\epsilon$ is sufficiently small, most particles will remain on closed curves (called KAM tori) that are only slight perturbations of the original streamlines. However, some streamlines break up, in accordance with the PoincaréBirkhoff theorem, to form chains of 'islands' bounded by KAM tori which are surrounded by chaotic trajectories. Within each island the same types of structure occur in a self-similar manner. As the perturbation increases, KAM tori break up to form Cantor sets called cantori, and the chaotic regions increase in size. For even larger perturbations, all KAM tori are destroyed.

The KAM theorem is explained in detail in Lichtenberg \& Lieberman (1992); a simple overview can be found in Tabor (1989). Since Aref's famous paper on chaotic 


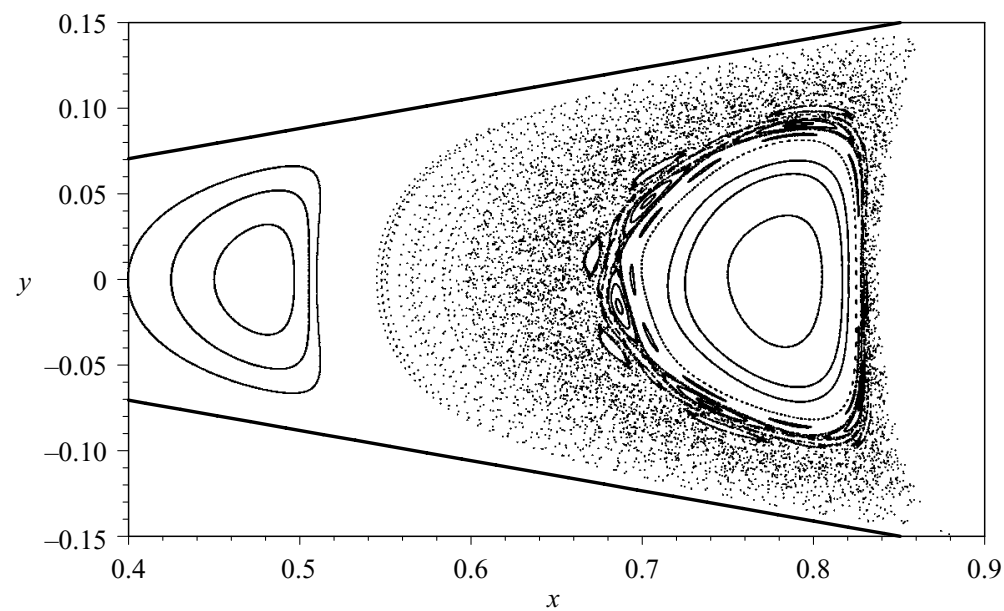

Figure 2. A Poincare section of particle motion in the perturbed flow; $2 \phi_{0}=20^{\circ}, K=1 / 2 \pi$ and $\epsilon=0.001$. Eighteen initial conditions have been evolved, eight of which are the original initial conditions for $\epsilon=0$, while another ten have been chosen for further detail; each regular trajectory is composed of 2000 points.

advection (Aref 1984), KAM and Poincaré-Birkhoff fixed-point theorems have found many uses in describing the breakup of particle paths into self-similar structures within Lagrangian fluid mechanics. For larger perturbations, this geometry disintegrates to chaotic advection of particles as can be witnessed, for example, within a fixed rectangular cavity at moderate Reynolds numbers, which has been described by Ottino (1989) and Horner et al. (2002).

Figures 2 to 4 show Poincare sections of particle trajectories, which are constructed by plotting particle positions at $t=0,1,2, \ldots, 1999$. Each figure shows several trajectories. The equations of motion have been integrated numerically using an explicit fourth-order Runge-Kutta scheme with a step size of 0.01 ; there is no obvious change in the figures when the step size is halved. Figures 2 and 3 show how the trajectories change as $\epsilon$ is increased from 0.001 to 0.5 for a corner with $2 \phi_{0}=20^{\circ}$. For $2 \phi_{0}=60^{\circ}$, figure 4 shows the qualitative differences between $\epsilon=0$ and $\epsilon=0.1$. Within the assumptions of this study, these examples are qualitatively typical of particle transport in a moving corner. As the parameter values for these illustrations are representative, attention will be restricted to describing the nature of particle motion occurring there. The interpretation of these figures is discussed in the next section.

\section{Discussion}

Before examining the qualitative nature of particle paths for $\epsilon \neq 0$, it is helpful to consider the unperturbed motion shown in figure 1 . Here the corner angle is $2 \phi_{0}=20^{\circ}$. Based on Moffatt's analysis of relative intensities, the left-hand eddy is around 380 times weaker than the right-hand eddy. So, by the time it takes a particle near the middle of the left-hand eddy to complete a circuit, one near the middle of the right-hand eddy has completed many circuits. This is reflected in the trajectory output for particles. For the left-hand eddy, each successive point on the path plotted is close to the previous point and the closed orbit is slowly traced out over time. For the right-hand eddy, successive points have a much larger gap between them and the particle completes many circuits before the orbit appears to be closed. So, particles in 

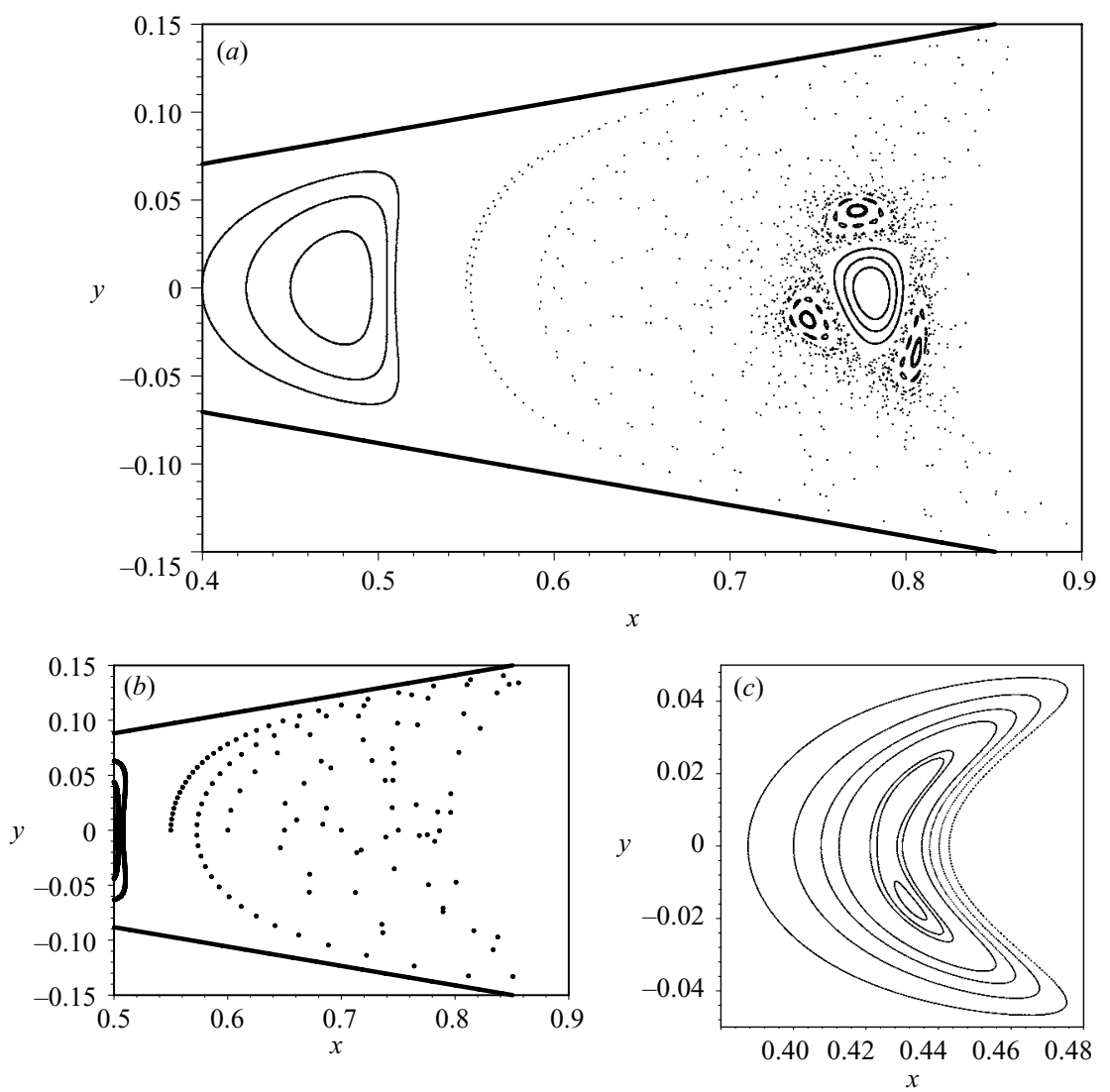

Figure 3. (a) A Poincaré section of particle motion in the perturbed flow; $2 \phi_{0}=20^{\circ}, K=1 / 2 \pi$ and $\epsilon=0.01$. Fourteen initial conditions have been evolved, eight of which are the original initial conditions for $\epsilon=0$, while another six have been chosen for further detail; each regular trajectory is composed of 2000 points. $(b, c)$ A Poincare section of particle motion in the perturbed flow; $2 \phi_{0}=20^{\circ}$ and $K=1 / 2 \pi$; each regular trajectory is composed of 2000 points. (b) Eight initial conditions have been evolved with $\epsilon=0.1$. The right-hand eddy is the focus of the view; the left-hand eddy has not changed qualitatively. (The points have been enlarged for clarity.) (c) Seven initial conditions have been evolved with $\epsilon=0.5$, three of which are the original initial conditions of the left-hand eddy for $\epsilon=0$, while another four have been chosen for further detail. The left-hand eddy is the focus of the view; the right-hand eddy has been destroyed.

the eddies circulate at different rates - this can be seen in figure 1 . The speed of the five particles for the right-hand eddy is represented by the highest five curves, while the lowest line represents the displacement of the three particles for the left-hand eddy (coinciding here for this scale). The periods illustrate that the particles of the left-hand eddy circulate, relatively speaking, slower than those on the right-hand eddy. Moreover, when considering each eddy separately, the closer a streamline is to the centre of the eddy, the shorter time it takes for a particle to complete a circuit of the closed curve relating to this streamline.

We now examine what happens when $\epsilon \neq 0$. Figure 2 shows a Poincaré section of some particle trajectories when $\epsilon=0.001$. The central part of the right-hand eddy behaves exactly as the KAM and the Poincaré-Birkhoff theorems suggest; several closed curves remain intact and chains of islands can be seen. However, trajectories 

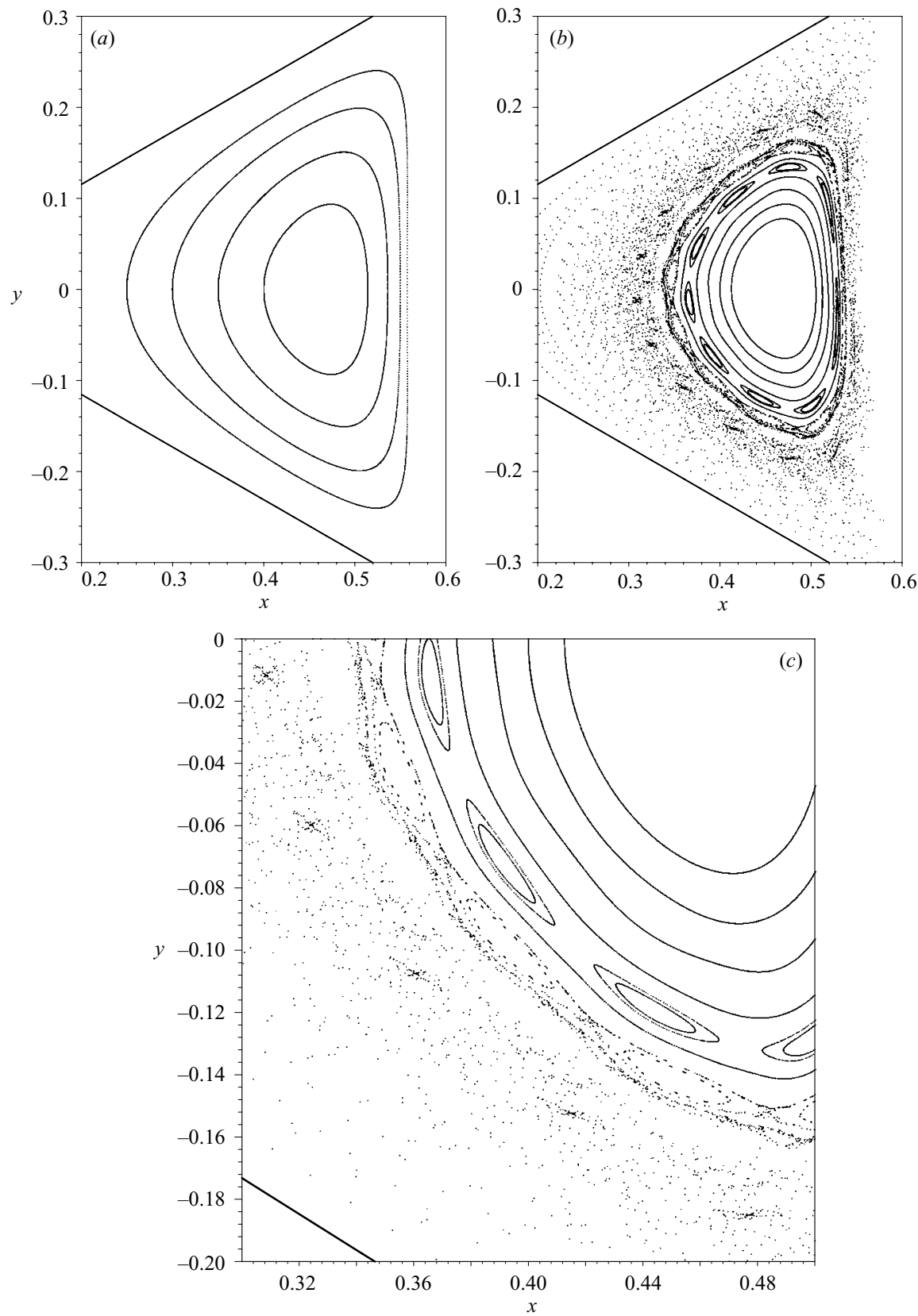

Figure 4. $(a, b)$ A Poincaré section of particle motion when $2 \phi_{0}=60^{\circ}$ and $K=1 / 2 \pi$; each regular trajectory is composed of 2000 points. (a) Four initial conditions have been evolved with $\epsilon=0$. (b) Fourteen initial conditions have been evolved with $\epsilon=0.1$, four of which are the original initial conditions for $\epsilon=0$, while another ten have been chosen for further detail. (c) An enlarged view of the bottom left-hand region of $(b)$.

in the outer part of the eddy are chaotic. By contrast, the Poincare section for the left-hand eddy appears to be unaffected by the perturbation.

Figure $3(a, b)$ shows the effects of increasing $\epsilon$ to 0.01 and 0.1 respectively. The righthand eddy behaves as expected: the KAM tori break up, so that the chaotic region

\section{CAmbridge JDURNALS}


increases in size. The left-hand eddy remains apparently unaffected. Indeed even if $\epsilon$ is increased to 0.5 , as in figure $3(c)$, many particles in the left-hand eddy seem to be moving around closed curves. In fact, the outer orbits have disintegrated, but the intermediate ones are merely deformed into 'cashew' shapes. The innermost ones lie on closed curves in the upper or lower central regions of the cashew (the figure shows one such curve in the lower region).

For a resting corner angle of $2 \phi_{0}=60^{\circ}$, the relative intensity for consecutive eddies will now be much larger. The smaller of a pair of neighbouring eddies is around 685 times weaker than its larger neighbour. Figure 4(a) shows unperturbed $(\epsilon=0)$ particle paths for one eddy, while figure $4(b, c)$ shows some trajectories for $\epsilon=0.1$. The trajectories behave similarly to those in the right-hand eddy in figure 2.

To help with identifying why there is a difference in behaviour between neighbouring eddies, let us return to the example with resting corner angle of $20^{\circ}$. With each eddy, the period depends continuously on the streamfunction. So it is not the case that none of the orbits in the weaker (left) eddy are resonant with the perturbation. The other possibility is that the resonances are very high order. Consider $\epsilon=0.001$; here the circulation is weaker (compared with $\epsilon=0$ ), indicating that this is the case. (Quasi-steady Stokes flows exhibiting lack of breakup of invariant curves also occur in Kaper \& Wiggins 1993, for instance.) When $\epsilon \nless 1$, the KAM theorem is no longer applicable. However, it is possible to account for other observed behaviour, as follows.

The left-hand eddy is not chaotic, because within it the Moffatt corner flow is a perturbation to the back-and-forth motion produced by the squeeze flow. A fluid particle moves into and out of the corner during any cycle, finishing very close to where it started. The difference in position is due to the very weak rotation produced by the Moffatt eddy. Increasing the strength of the squeeze flow does not change the Poincare section significantly, unless the squeeze flow is so great that particles are pushed into regions where they can experience strong Moffatt eddies. This explains why the left-hand eddy appears unchanged unless $\epsilon$ is large.

From these results we can infer that there is an optimal region for chaotic transport in a corner. By 'chaotic transport' we mean that the local Lyapunov exponent is positive. For large or strong Moffatt eddies (in the sense of the relative scale and intensity factors calculated by Moffatt), the wall motion provides only a weak perturbation, so the island chains are thin. For small or weak Moffatt eddies, the rotation is a perturbation to the motion produced by the squeeze flow. In either of these cases, most trajectories are regular. Chaotic transport is maximized in the region where the squeeze flow is a moderate perturbation to the Moffatt flow.

To support the hypothesis that there is a region where chaotic transport is maximized, consider the next furthest eddy from the corner. Figure 5(a) shows such an eddy; the step size is 0.0001 . This is necessarily smaller than for the previous figures so that the very fast moving particles of the largest eddy can be adequately tracked, especially near its right-hand separatrix. In figure $5(b)$, perturbing the walls shows the disintegration of its left-hand neighbour (compare with figure 3 ). The particle trajectories of the largest eddy that lie sufficiently far from the walls appear robust to the perturbation. This is expected because at this distance from the corner the squeeze flow is a very weak perturbation to the strong rotation of the Moffatt eddy. Figure $5(c)$ shows that some particles in the largest eddy lie on KAM tori in thin islands, as predicted by KAM theory. The thinness of these islands is due to the weakness of the squeeze flow perturbation.

The model examined in this study only focuses on the effect of wall motion on recirculating particles (to leading order for both the Moffatt flow and the squeeze 

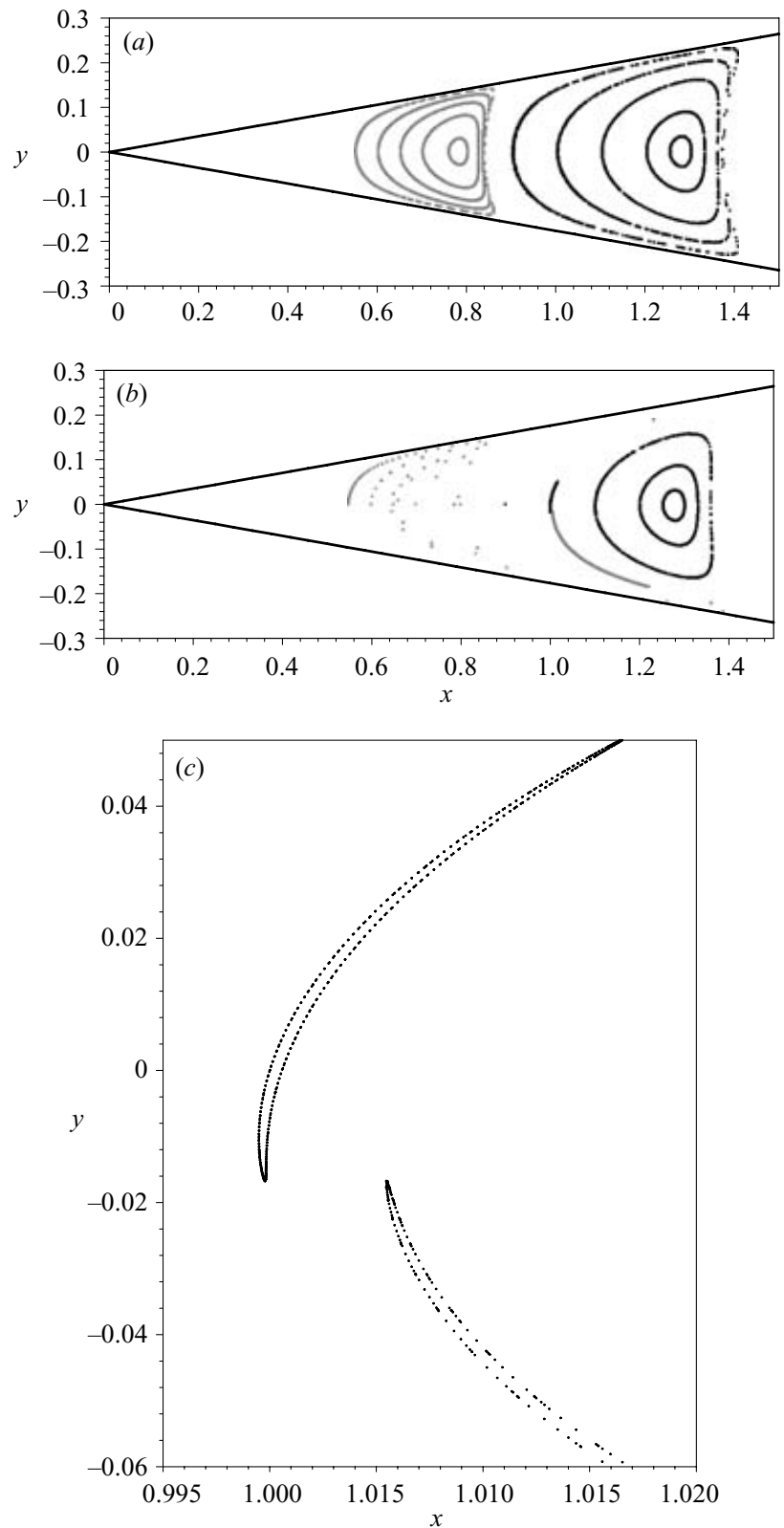

FiguRE 5. A Poincaré section of particle motion when $2 \phi_{0}=20^{\circ}$ and $K=1 / 2 \pi$; each regular trajectory is composed of 500 points. Ten initial conditions have been evolved. (The points have been enlarged for clarity.) $(a)$ Fixed walls $(\epsilon=0)$. (b) Moving walls with $\epsilon=0.1$. (c) A closer look at $(b)$.

flow). Even so, it has shown that just with very simple boundary conditions, the cyclic expansion and contraction causes passive particles to exhibit chaotic motion in very low-Reynolds-number flow.

It is reasonable to ask whether our two-dimensional model gives any insight into real alveolar flows, which are three-dimensional. Provided that an alveolar cavity is sufficiently deep, recirculation has been shown to occur; Tsuda et al. (2002). Our

\section{CAmbridge JDURALS}


results suggest that in places where the squeeze flow driven by the wall motion is of the same order of magnitude as recirculation in the centre, some particles move chaotically. It is unclear whether or not such motion occurs at the edges of the alveoli.

The moving-corner model may be extended to incorporate diffusion and particle inertia. Furthermore, the far-field motion can be adjusted so that $K$ is time-dependent and incorporates periodic motion (as well as a time lag). These investigations will be presented elsewhere.

\section{Conclusions}

We have shown that, qualitatively speaking, particle transport in a moving-walled corner occurs in two distinct ways. In eddies near to the corner the wall motion dominates, and the Moffatt eddies provide a weak perturbation. However, this weak perturbation drives an average particle motion similar to the motion produced by the steady eddy. Away from the corner the far-field motion dominates, encouraging islands of KAM tori inside each other (as in figure $3 a$ ) and chaotic trajectories to form within a limited region, thereby enhancing mixing of particles. To the best of our knowledge, this is the first clear theoretical indication that in small airways, where the Reynolds number is very low, there is a mechanism for producing chaos.

F.E. L.-P. and P.E.H. are supported by the NIH under grant number BRP HL070542. This is a collaborative project led by Akira Tsuda of the Harvard School of Public Health.

\section{REFERENCES}

ACheson, D. J. 1990 Elementary Fluid Dynamics. Oxford University Press.

AreF, H. 1984 Stirring by chaotic advection. J. Fluid Mech. 143, 1-21.

Chetan, P. M., Weidman, P. D. \& Davis, A. M. J. 2005 Nested toroidal vortices between concentric cones. J. Fluid Mech. 522, 117-139.

Haber, S., Butler, J. P., Brenner, H., Emanuel, I. \& Tsuda, A. 2000 Shear flow over a self-similar expanding pulmonary alveolus during rhymical breathing. J. Fluid Mech. 405, 243-268.

Henry, F. S., Butler, J. P. \& Tsuda, A. 2002 Kinematically irreversible acinar flow: a departure from classical dispersive aerosol transport theories. J. Appl. Physiol. 92, 835-845.

Horner, M., Metcalfe, G., Wiggins, S. \& Ottino, J. M. 2002 Transport enhancement mechanisms in open cavities. J. Fluid Mech. 452, 199-229.

KAPER, T. J. \& WigGins, S. 1993 An analytical study of transport in Stokes flows exhibiting large-scale chaos in the eccentric journal bearing. J. Fluid Mech. 253, 211-243.

Lichtenberg, A. J. \& Lieberman, M. A. 1992 Regular and Chaotic Dynamics, 2nd edn. Springer.

LiU, C. H. \& JosePH, D. D. 1978 Stokes flow in conical trenches. SIAM J. Appl. Maths 34, 286-296.

Moffatt, H. K. 1964 Viscous and resistive eddies near a sharp corner. J. Fluid Mech. 18, 1-18.

Moffat, H. K. \& Duffy, B. R. 1980 Local similarity solutions and their limitations. J. Fluid Mech. 96, 299-313.

Ottino, J. M. 1989 The Kinematics of Mixing: Stretching, Chaos, and Transport. Cambridge University Press.

Shankar, P. N. 2000 On Stokes flow in a semi-infinite wedge. J. Fluid Mech. 422, 69-90.

TABOR, M. 1989 Chaos and Integrability in Nonlinear Dynamics. Wiley.

TANEDA, S. 1979 Visualization of separating Stokes flows. J. Phys. Soc. Japan 46, 1935-1942.

Tsuda, A., Henry, F. S. \& ButLer, J. P. 1995 Chaotic mixing of alveolated duct flow in rhythmically expanding pulmonary acinus. J. Appl. Physiol. 79, 1055-1063.

Tsuda, A., Rogers, R. A., Hydon, P. E. \& Butler, J. P. 2002 Chaotic mixing deep in the lung. Proc. Natl Acad. Sci. 19, 10173-10178.

Weidman, P. D. \& CALmidi, V. 1999 Instantaneous Stokes flow in a conical apex aligned with gravity and bounded by a stress-free surface. SIAM J. Appl. Maths 59, 1520-1531. 